\title{
GEOGRAFIA E REPRESENTAÇÕES SOCIAIS: ESTADO DA ARTE
}

\author{
STATE OF THE ART OF RESEARCHES RELATED TO GEOGRAPHY \\ AND SOCIAL REPRESENTATIONS \\ ÉTAT DE L'ART DES RECHERCHES QUI CONCERNENT GEOGRAPHIE \\ ET DES REPRÉSENTATIONS SOCIALES
}

\section{Aline Camilo Barbosa}

Mestre em Geografia pelo Programa de Pós-graduação em Geografia da Universidade Federal do Piauí (PPGGEO-UFPI). Endereço: Endereço: Rua Altos, n 5551, Bairro Alto Alegre - CEP 64006-160. Teresina/PI. E.mail: alinecamilo_barbosa@hotmail.com

\section{Josélia Saraiva Silva}

Doutora em Educação pela Universidade Federal do Rio Grande do Norte. Docente no Programa de Pós-graduação em Geografia da Universidade Federal do Piauí (PPGGEO-UFPI). Endereço: Campus Ministro Petrônio Portella, Bloco do CCE, sala 06. Bairro Ininga-CEP: 64049-550. Teresina/PI. E.mail: joseliasaraiva@yahoo.com.br

\section{RESUMO}

Neste artigo apresentamos o Estado da Arte das pesquisas que relacionam Geografia e a Teoria das Representações Sociais desenvolvida pelo pesquisador francês Serge Moscovici. Analisamos dissertações e teses disponíveis em dois bancos de dados, produzidas no período de uma década (2005-2015). O objetivo foi evidenciar como as pesquisas de Geografia utilizam o aporte teórico das representações sociais. Desse modo, buscamos conhecer os objetos representacionais investigados, os instrumentos de coleta de dados utilizados e, ainda, o uso de teoria complementar. Além disso, nesse estudo, foi possível visualizar em quais universidades brasileiras essas pesquisas foram realizadas e como se dá a sua espacialização no território nacional. Para a análise, organizamos dois grupos temáticos: o ensino de geografia e as representações sociais e; a ciência geográfica e as representações sociais. Como resultado, pudemos constatar um crescente aumento ao longo dos anos das produções que buscam essa aproximação, contudo, esses números são ainda incipientes, demonstrando que o uso da teoria das representações sociais nas pesquisas de Geografia é um campo a ser explorado.

Palavras - chave: Estado da Arte; Representações Sociais; Geografia; Produção Acadêmica. 


\begin{abstract}
In this article we present the State of the Art of the research that relates Geography and the Theory of Social Representations developed by the French researcher Serge Moscovici. We analyzed dissertations and theses available in two databases, produced over a decade (2005-2015). The objective was to show how Geography researches use the theoretical contribution of social representations. In this way, we seek to know the representational objects investigated, the instruments used and if there is the use of complementary theory. In addition, in this study, it was possible to visualize in which Brazilian universities these researches were carried out and how their spatialization occurs in the national territory. For the analysis, we organized two thematic groups: the teaching of geography and the social representations and; Geographical science and social representations. As a result, we can see a growing increase over the years of productions that seek this approach, however, these numbers are still incipient, demonstrating that the use of the theory of social representations in geography research is a field to be explored.
\end{abstract}

Key words: State of Art; Social Representations; Geography; Academic Production.

\title{
RÉSUMÉ
}

Dans cet article, nous présentons l'état de l'art des recherches reliant la géographie et la théorie des représentations sociales développés par le chercheur français Serge Moscovici. Nous avons analysé des dissertations et thèses disponibles dans deux databases, produites dans la période de dix ans (2005-2015). Lobjectif était de démontrer comment les recherches dans la géographie utilisent le cadre théorique des représentations sociales. Ainsi, nous avons cherché à connaître les objets représentatifs étudiés, les instruments utilisés et s'il y a eu l'utilisation de la théorie complémentaire. En outre, dans cette étude, il était possible de voir dans lequelles universités brésiliennes telles recherches ont été faites et comment est leur répartition spatiale dans le pays. Pour l'analyse, nous avons organisé deux groupes thématiques: l'enseignement de la géographie et des représentations sociales et; la science géographique et les représentations sociales. En conséquence, nous avons constaté une augmentation constante au cours des années de productions qui cherchent cette approche, cependant, ces chiffres sont encore incomplètes, ce qui démontre que l'utilisation de la théorie des représentations sociales dans la recherche géographique est un domaine à explorer.

Mots-clés: état de l'art; Les représentations sociales; la géographie; production académique. 


\section{INTRODUÇÃO}

Em uma pesquisa realizada por Martins et. al.(2014), que teve como objetivo identificar os grupos de pesquisas brasileiros cadastrados no Conselho Nacional de Pesquisa e Desenvolvimento Tecnológico - CNPq, que desenvolvem investigações com o uso da teoria da Representação Social, o campo da educação aparece como destaque; constatou-se que do total absoluto de 172 grupos cadastrados, 50 são da área de educação, correspondendo a 49\%. Ao analisar especificamente o contexto da Geografia, observamos um número pequeno de grupos, o que nos revela que nesta ciência têm-se poucas pesquisas com uso dessa teoria.

De acordo com Bomfim (2012, p. 23), o termo Representação Social aparece para a ciência geográfica nos anos de 1980, nos trabalhos de Guerin (1985), Gummuchian (1985; 1989) e Bailly (1995) “[...] que afirmam que a Geografia consiste nas representações de objetos, de práticas e de processos espaciais graças aos conceitos evolutivos”. Para Cavalcanti (1998), as representações sociais são recursos importantes para compreender que concepções, ideias, conceitos e imagens sobre Geografia as crianças e adolescentes estão formando nos diferentes ambientes, como também no âmbito escolar. Além disso, podem indicar caminhos para melhor compreender essa ciência.

Assim, com o objetivo de identificar o uso do aporte teórico das representações sociais nas pesquisas da ciência geográfica optamos por elaborar um estado da arte. Esse tipo de investigação possibilita esquematizar como esse conhecimento vem sendo desenvolvido nas pesquisas científicas nas universidades públicas brasileiras, além disso, esse tipo de pesquisa pode orientar o leitor que possui interesse por esta temática, e que deseje trabalhar com a referida teoria.

\section{CAMINHOS DA INVESTIGAÇÃO}

Neste artigo apresentamos o resultado de uma investigação sobre dissertações e teses de Geografia que utilizam como aporte teórico metodológico a Teoria das Representações Sociais desenvolvida por Serge Moscovici.

O presente estudo tem suporte nas pesquisas que se utilizam do aporte metodológico: estado da arte. De acordo com Ferreira (2002) esse tipo de pesquisa tem caráter bibliográfico e objetiva mapear as produções acadêmicas de um determinado conhecimento, podendo, portanto, ser utilizado em todas as áreas do conhecimento. Esse levantamento, ao ser realizado, possibilita responder questões sobre o modo como vem sendo produzido esse conhecimento; em que período foi mais privilegiado, que público é investigado, entre outros (FERREIRA, 2002). 
Para Fonseca, Salvi e Torres (2014, p. 143) o estado da arte é “[...] uma atividade árdua e complexa, justamente por seu caráter crítico e reflexivo. Auxilia na otimização e também no desenvolvimento de novas demandas de pesquisa, conceitos e paradigmas". Esses aspectos estão associados ao grande número de pesquisas na área de educação, porém, poucas na área de geografia, as quais nos fizeram optar por esse tipo de pesquisa tendo como escopo a Teoria das Representações Sociais. Consideramos que essa teoria pode auxiliar na compreensão da ciência geográfica como conhecimento compartilhado na sociedade.

Para organizar essa pesquisa, a primeira fase foi eleger um banco de informações nacional para realizar a busca dos dados. Optamos pela Biblioteca Digital Brasileira de Teses e Dissertações - BDTD e o banco de dados do Programa de Pós - Graduação em Geografia da Universidade Federal do Piauí-PPGGEOํ. O período estabelecido foi de uma década variando de 2005 a 2015.

A consulta com relação ao BDTD foi realizada durante todo o mês de junho de 2016, em que utilizamos a opção busca avançada com o uso dos termos: "representação social e geografia" e; "representações sociais e geografia", sendo presente em todos os campos: assunto, instrumentos, títulos, resumos e palavras-chave. Com essa investigação, detectamos um total de 307 trabalhos que apresentavam esses termos, sendo "representação social e geografia" em 149 trabalhos e "representações sociais e geografia" em 158 pesquisas.

Com relação ao banco de dados do PPGGEO, também fizemos a pesquisa no banco de dados do seu sistema on-line no mês de junho/2016 e encontramos um total de 9 trabalhos em que estava presente o termo "representações sociais e geografia".

Durante a análise, organizamos os trabalhos coletados em dois grupos temáticos considerando a origem dos objetos estudados: Ensino de geografia e representações sociais; e ciência geográfica e representações sociais. Estabelecemos como critérios de análise os seguintes tópicos que foram aplicados a cada um desses grupos: aplicação da teoria das representações sociais e abordagens complementares; objetos representacionais (aqueles dos quais foram construídas representações sociais); a metodologia de pesquisa utilizada; o volume de produção anual e sua espacialização no Brasil.

Para prosseguirmos na análise, consideramos pertinente apresentar, inicialmente, os pressupostos da Teoria da Representação Social de Serge Moscovici e suas abordagens complementares (Jean - Claude Abric, Denise Jodelet e Willen Doise).

\footnotetext{
1 Ao realizar uma pesquisa na Biblioteca Digital Brasileira de Teses e Dissertações, não encontramos informações referentes ao Programa de Pós-graduação em Geografia da Universidade Federal do Piauí-PPGGEO. Consideramos importante a presença dos dados deste Programa em nossa pesquisa em virtude de que este possui uma linha de pesquisa de ensino de Geografia e contar com pesquisadores que utilizam a Teoria das Representações Sociais em suas investigações. Desse modo, optamos por inserir o seu banco de dados na pesquisa.
} 


\section{REPRESENTAÇÕES SOCIAIS: FENÔMENOS COTIDIANOS}

As Representações Sociais são fenômenos complexos que devem ser compreendidos dentro de um contexto social. Para Moscovici (2012, p. 27) devem ser percebidas como “[...] uma modalidade de conhecimento particular tendo a função de elaboração dos comportamentos e da comunicação entre os indivíduos" (grifo do autor). Ou seja, apresenta-se como um corpo de conhecimento organizado que advém da troca de informações entre os indivíduos, que produz e determina comportamentos nos diferentes grupos.

Com relação ao surgimento da ideia de representações sociais, Moscovici (2012) atribuiu a Durkheim como o primeiro a perceber que as produções mentais poderiam ser tanto individuais como sociais. Embasado nessa visão, esse sociólogo estabeleceu as representações individuais e coletivas: as primeiras relacionavam-se com o campo da Psicologia, tendo por substrato a consciência de cada um, enquanto a última estava ligada ao campo da Sociologia, considerando a sociedade em sua totalidade (MOSCOVICI, 2003; 2001).

Partindo das representações coletivas de Durkheim, Moscovici (2012) elabora a ideia de representações sociais. A diferença entre essas representações centra-se na abordagem. Para Durkheim, as representações coletivas expressavam um caráter engessado, consideradas como “[...] formas estáveis de compreensão coletiva [...]" (MOSCOVICI, 2003, p. 15); já Moscovici (2003) demonstra que as Representações Sociais tinham caráter social, podendo incluir conceitos psicológicos, como também sociológicos, indicando assim, maior plasticidade, explorando a variação e a diversidade de ideias coletivas na sociedade.

A obra seminal dessa Teoria foi a tese de doutorado de Serge Moscovici, sendo relatada inicialmente no livro La psychanalyse, Son image et son public, publicado na França no ano de 1961. Esta Teoria tinha como projeto a construção de uma Psicologia Social, que se caracterizava como uma tentativa de unir ideias das ciências Psicologia e Sociologia. Entre os propósitos da Teoria de Moscovici, temos a busca pela relação entre indivíduo e sociedade na constituição do conhecimento em meio ao grupo social.

A difusão da teoria ocorre desde então e vem sendo alvo de trabalhos de pesquisadores em várias partes do mundo. Essas pesquisas aperfeiçoam e aprofundam a teoria de tal sorte que é possível observar a existência de abordagens complementares a ela. Destacamos os trabalhos de Denise Jodelet, Jean-Claude Abric e Willem Doise que, segundo Sá (1998), são as mais exploradas nos trabalhos brasileiros dedicados a essa teoria. Dessa maneira, iremos expô-las brevemente. 
Denise Jodelet (2001) foi uma das principais colaboradoras de Moscovici, buscando em sua abordagem conceituar as representações sociais, contribuindo diretamente com a “Grande Teoria”. Para Jodelet (2001, p. 22) a representação social “[...] é uma forma de conhecimento, socialmente elaborado e partilhado, com um objeto prático, e que contribui para a construção de uma realidade comum a um conjunto social”.

Enfatizando o caráter de construção social, Jodelet destaca que as representações sociais podem ser observadas na relação dos grupos com determinados objetos sociais, havendo, portanto, uma relação simbólica e de interpretação. Assim, as representações são sempre uma forma de saber ou de conhecer, o que impulsiona a ação dos sujeitos sobre o mundo.

Dessa maneira, Jodelet (2001, p. 28) apresenta um viés descritivo e enfatiza que para dá conta dos elementos que envolvem as representações é necessário responder algumas perguntas: "Quem sabe e de onde sabe?”; “O que e como sabe?”; "Sobre o que sabe e com que efeitos?" Dessa forma, sua pesquisa tem base empírica, vinculadas nos discursos das pessoas e dos grupos, no comportamento e nas práticas.

Com relação a Jean-Claude Abric (2001), este apresenta uma perspectiva estruturalista. Desenvolveu a Teoria do Núcleo Central (TNC). Segundo essa teoria, as representações sociais são compostas de elementos centrais e periféricos. Os elementos centrais são os mais estáveis da representação e que resistem às mudanças; os elementos periféricos já permitem mudanças, ou seja, são flexíveis. Nessa ótica, para essa perspectiva o elemento principal da representação é o sistema central, visto que “[...] é ele que determina ao mesmo tempo sua significação e sua organização” (ABRIC, 2001, p. 163).

Na perspectiva de Willem Doise, as representações sociais são “[...] princípios geradores de tomada de posição ligadas a inserções específicas em um conjunto de relações sociais e que organizam os processos simbólicos que intervém nessas relações" (DOISE,1990, p. 125 citado por SÀ, 1996, p. 74-75). Esse autor enfatiza que a interação e a inserção dos indivíduos ao grupo são importantes para a constituição das representações. Indica que sua perspectiva de representação social está vinculada as ideias do sociólogo francês Pierre Bourdieu.

Segundo Sá (1998), as ideias de Doise estão ligadas, principalmente, à ancoragem elaborada por Serge Moscovici, processo que dá sentido ao objeto representado. Partindo dessa perspectiva, a ancoragem, para Doise, sempre se constitui e opera no âmbito de uma determinada posição no campo social, ao apontar isso, o autor foca suas análises nos aspectos sociológicos da representação. 
Essas são três perspectivas possíveis para melhor analisar as representações sociais. A escolha de cada uma vai se dá a partir da definição dos objetivos de cada pesquisa. Feitas essas considerações, seguimos a análise de como essa Teoria vem se desenvolvendo nas pesquisas brasileiras de Geografia.

\section{PRODUÇÃO ANUAL E SUA ESPACIALIZAÇÃO NO BRASIL: INICIANDO A ANÁLISE}

Um aspecto importante de ser observado é o fato de que há um crescimento no volume de produção ao longo do período em análise.

Tabela 1- Volume de publicações anual nos dois grupos temáticos (2005-2015)

\begin{tabular}{|c|c|c|c|c|c|c|c|c|c|c|c|}
\hline Anos & 2005 & 2006 & 2007 & 2008 & 2009 & 2010 & 2011 & 2012 & 2013 & 2014 & 2015 \\
\hline $\begin{array}{l}\text { Ensino de geografia e } \\
\text { representações sociais }\end{array}$ & 1 & 3 & 1 & 2 & 0 & 2 & 0 & 4 & 1 & 4 & 4 \\
\hline $\begin{array}{l}\text { Ciência geográfica e } \\
\text { representações sociais }\end{array}$ & 2 & 1 & 0 & 1 & 3 & 3 & $3^{2}$ & 1 & 5 & 3 & 3 \\
\hline Variações de anos & \multicolumn{5}{|c|}{$2005-2010$} & \multicolumn{6}{|c|}{$2011-2015$} \\
\hline Total das variações & \multicolumn{5}{|c|}{19} & \multicolumn{6}{|c|}{28} \\
\hline
\end{tabular}

Fonte: BNTD (2016)

Organizado: Barbosa (2016)

A tabela 1 apresenta o número de produções por ano, tendo a variação de 2005 a 2015. Com relação ao grupo temático de ensino de Geografia e representações sociais, realizando um paralelo, temos a seguinte informação: da variação de 2005 a 2010 temos um total de 9 (nove) produções e; de 2011 a 2015 temos 13 (treze) produções. Esses dados mostram que nesses últimos anos os trabalhos de ensino de Geografia que utilizam o aporte teórico das representações sociais têm aumentado em relação aos primeiros anos, o que nos infere dizer que os pesquisadores têm se interessado pelas contribuições da teoria em destaque nas pesquisas de Ensino de Geografia.

Com respeito ao grupo temático da ciência geografia e representações sociais, foi apresentado de 2005 a 2015 um número de 10 (dez) produções e; 2011 a 2015 um número de 15 (quinze) publicações. Assim, consideramos que esse grupo temático também teve aumento de produção em relação às variações de anos.

\footnotetext{
No ano de 2011 foram selecionados três trabalhos, contudo, uma das pesquisas não foi encontrada. O link que corresponde a esse trabalho na Biblioteca Digital Brasileira de Teses e Dissertações - BDTD não abre o documento, mesmo realizando pesquisas na internet por meio do seu título também não conseguimos localizar essa pesquisa. Assim, nas análises que serão realizadas a seguir não iremos contar com essa produção.
} 
Ao analisar os dados com ênfase na temporalidade, constatamos que os trabalhos com ênfase no ensino e geográfico ao longo da variação 2005 a 2015 tem tido um relativo aumento de produção se comparado com a ciência geográfica. Acreditamos que esse interesse se dá pela relevância que a representação social trás nas pesquisas de ensino, visto que essa teoria nos possibilita visualizar como os diferentes públicos compartilham ideias e visões sobre um determinado objeto.

Observando esse volume crescente de produção, buscamos espacializa-lo para vermos como ocorre esse desenvolvimento ao longo do território nacional.

Tabela 2 - Espacialização das pesquisas em geografia e representação social no Brasil (2005-2015)

\begin{tabular}{|c|c|c|c|c|}
\hline GRUPOS TEMÁTICOS & REGIÃO & $\begin{array}{l}\text { UNIDADE DA } \\
\text { FEDERAÇÃO }\end{array}$ & $\begin{array}{l}\text { INSTITUIÇÃO } \\
\text { PÚBLICA }\end{array}$ & $\begin{array}{l}\text { QUANTIDADE } \\
\text { DE PUBLICAÇẪO }\end{array}$ \\
\hline \multirow{11}{*}{$\begin{array}{l}\text { Ensino de geografia e } \\
\text { representações sociais }\end{array}$} & \multirow{2}{*}{ Sul } & RS & UFRGS & 6 \\
\hline & & PR & UFPR & 1 \\
\hline & \multirow{2}{*}{ Sudeste } & $\mathrm{SP}$ & USP & 1 \\
\hline & & $\mathrm{SP}$ & UNESP & 1 \\
\hline & \multirow{4}{*}{ Nordeste } & $\mathrm{PI}$ & UFPI & 5 \\
\hline & & $\mathrm{RN}$ & UFRN & 2 \\
\hline & & PB & UFPB & 1 \\
\hline & & MA & UFMA & 1 \\
\hline & \multirow{3}{*}{ Centro-oeste } & DF & UNB & 1 \\
\hline & & MG & UFU & 1 \\
\hline & & $\mathrm{GO}$ & UFG & 2 \\
\hline TOTAL & & & & 22 \\
\hline \multirow{12}{*}{$\begin{array}{l}\text { Ciência geográfica e } \\
\text { representações sociais }\end{array}$} & \multirow{3}{*}{ Sul } & RS & UFRGS & 1 \\
\hline & & PR & UFPR & 4 \\
\hline & & SC & UFSC & 1 \\
\hline & \multirow{4}{*}{ Sudeste } & SP & USP & 1 \\
\hline & & SP & UNESP & 5 \\
\hline & & MG & UFMG & 1 \\
\hline & & $\mathrm{RJ}$ & PUC & 1 \\
\hline & \multirow{3}{*}{ Nordeste } & $\mathrm{PI}$ & UFPI & 2 \\
\hline & & SE & UFS & 2 \\
\hline & & PB & UFPB & 1 \\
\hline & \multirow{2}{*}{ Centro-oeste } & DF & UNB & 4 \\
\hline & & MS & UFMS & 1 \\
\hline TOTAL & & & & 24 \\
\hline
\end{tabular}

Fonte: BNTD (2016)

Organizado: Barbosa (2016) 
A tabela 2 mostra que a maioria dos trabalhos de ensino de geografia e representações sociais ao longo de 2005 a 2015 foi produzida na região Nordeste, correspondendo a um total de 09 (nove) trabalhos com destaque para a produção do estado do Piauí que contou com 05 (cinco) trabalhos produzidos. Em segundo lugar vem a região Sul, com 07 (sete) trabalhos, sendo 6 (seis) produzidos pela Universidade Federal do Rio Grande do Sul e 1 (um) pela Universidade Federal do Paraná. A região Centro-oeste apresentou 4 (quatro) publicações, sendo 2 (duas) produções da Universidade Federal de Goiás, 1 (uma) na Universidade Federal de Uberlândia e 1 (uma) na Universidade de Brasília. Na região Sudeste tivemos a presença de dois trabalhos, 1 (um) na Universidade Estadual Paulista e 1 (um) na Universidade de São Paulo. Uma observação importante é que não encontramos produções desse grupo temático na região Norte do Brasil.

Com relação à espacialização dos trabalhos da área da ciência geográfica e representações sociais, a maior produção encontra-se na região Sudeste, com um total de 8 (oito) publicações, correspondendo a 5 (cinco) na Universidade Estadual Paulista e 1 (uma) na Universidade de São Paulo, Universidade Federal de Minas Gerais, e Pontifícia Universidade Católica do Rio de Janeiro.

Em seguida temos a região Sul, com 6 (seis) trabalhos, em sua maioria as publicações correspondem à Universidade Federal do Paraná com 4 (quatro) publicações. A Universidade Federal de Santa Catarina e a Universidade Federal do Rio Grande do Sul apresentam 1 (um) trabalho cada. Empatados com 5 (cinco) publicações cada, temos as regiões Centro-oeste e região Nordeste. A primeira com a Universidade de Brasília, com 4 (quatro) publicações e a Universidade Federal do Mato Grosso do Sul com 1(uma) produção. No Nordeste, temos a Universidade Federal do Piauí e a Universidade Federal de Sergipe com 2 (duas) produções cada e a Universidade Federal da Paraíba com 1 (um) trabalho. Com relação à região Norte do Brasil, também não encontramos produções desse grupo temático com uso da teoria em destaque.

Ao observarmos esses dados da espacialização, evidenciamos uma produção incipiente de estudos no campo da geografia, tendo como aporte teórico as representações sociais. Ressaltamos que essas pesquisas contribuem para o conhecimento de elementos psicossociais importantes para a análise da relação dos agentes sociais com o espaço geográfico. Um outro aspecto relevante é que esses dados podem contribuir com futuros pesquisadores que possuem interesse na área de representação social e geografia, isso porque através dessas, pode-se localizar as instituições de ensino superior que dará suporte e apoio às suas pesquisas. 


\section{APLICAÇÃO DA TEORIA DAS REPRESENTAÇ̃̃ES SOCIAIS E ABORDAGENS COMPLEMENTARES}

Como dissemos e fizemos anteriormente, para a análise dos dados, decidimos reunir os trabalhos em dois grupos temáticos: Ensino de geografia e representações sociais e ciência geográfica e representações sociais. Para uma melhor visualização, na tabela 3 temos o total de trabalho por grupo temático de análise.

Tabela 3- Distribuição das dissertações e teses pesquisadas por grupo temático.

\begin{tabular}{c|c|c|c} 
Grupos & Dissertações & Teses & Total $^{2}$ \\
\hline Ensino de geografia e representações sociais & 19 & 03 & 22 \\
\hline Ciência geográfica e representações sociais & 18 & 06 & 24 \\
\hline TOTAL GERAL & 37 & 9 & 46 \\
\hline
\end{tabular}

Fonte: BNTD (2016)

Organizado: Barbosa (2016)

A tabela 3 apresenta o número de trabalhos que foram localizados durante a pesquisa. No total foram 46 trabalhos, sendo 37 dissertações que correspondem a um percentual de $80,4 \%$, e 09 teses que perfazem 19,6\%. Verificamos que em todos os grupos acontece uma predominância do número de dissertações em relação às teses. Como primeiro tópico de análise, elegemos a aplicação da teoria das representações sociais e suas abordagens complementares. Para uma melhor organização dos dados, realizaremos o diagnóstico por grupo temático.

No que concerne ao grupo de ensino de geografia e representações sociais que contém 22 (vinte e dois) trabalhos, 16 (dezesseis) que correspondem a 72,7\% utilizam somente a Grande Teoria, elaborada por Serge Moscovici, enquanto os 6 (seis) trabalhos restantes $(27,3 \%)$ usam além da Grande Teoria, a abordagem complementar desenvolvida por JeanClaude Abric.

No grupo temático da ciência geográfica e representações sociais, que conta com 24 (vinte e quatro) trabalhos, 03 (três) deles (12,5\%) não utilizam a teoria das representações sociais desenvolvida por Serge Moscovici, visto que nestes não temos a presença de nenhuma referência bibliográfica conhecida que trate sobre a teoria. Contudo, temos nos textos a presença da expressão “representações sociais". Em relação às demais produções, 15 (quinze) trabalhos $(62,5 \%)$ utilizam somente a Grande Teoria e; 6 (seis) trabalhos (25\%) usam a Grande Teoria e a abordagem complementar da Teoria do Núcleo Central.

Número de dissertações mais o número de teses. 
Observando esses dados, podemos considerar que a maioria dos trabalhos utiliza a teoria das representações sociais, tendo como aporte teórico Serge Moscovici e dentre as abordagens complementares somente a Teoria do Núcleo Central foi privilegiada nessas pesquisas.

Com relação aos objetos representacionais constatamos que existe uma diversidade em todos os grupos temáticos. Por esse motivo, para esse estudo optamos por organizálos em categorias.

No grupo de ensino de geografia e representações sociais foi possível obtermos três categorias: a primeira foi processos educacionais que engloba trabalhos direcionados a temas pedagógicos. A segunda categoria são conceitos geográficos na qual estão colocados os trabalhos que têm como objeto representacional os conceitos-chave da geografia e; por fim, a categoria temas geográficos que apresenta temas variados presente no contexto da ciência geográfica. Para uma melhor visualização, temos a tabela 4.

Tabela 4- Categorização dos objetos representacionais do grupo temático ensino de geografia e representações sociais

\begin{tabular}{|c|c|c|c|}
\hline Categoria & $\begin{array}{l}\text { Frequência } \\
\text { ( } n^{\circ} \text { absoluto) }\end{array}$ & $\begin{array}{l}\text { Porcentagem } \\
(\%)\end{array}$ & $\begin{array}{l}\text { Objetos representacionais } \\
\text { (exemplos encontrados) }\end{array}$ \\
\hline \multirow{4}{*}{ Temas geográficos } & \multirow{4}{*}{11} & \multirow{4}{*}{50} & Cerrado \\
\hline & & & Continente Africano \\
\hline & & & Espaço urbano \\
\hline & & & Meio ambiente \\
\hline \multirow{4}{*}{ Processos educacionais } & \multirow{4}{*}{7} & \multirow{4}{*}{31,8} & Geografia escolar \\
\hline & & & Educação inclusiva \\
\hline & & & Avaliação de geografia \\
\hline & & & Ensinar geografia \\
\hline \multirow{4}{*}{ Conceitos geográficos } & \multirow{4}{*}{4} & \multirow{4}{*}{18,2} & Espaço \\
\hline & & & Lugar \\
\hline & & & Paisagem \\
\hline & & & Espaço geográfico \\
\hline Total & 22 & 100 & \\
\hline
\end{tabular}

Fonte: BNTD (2016)

Organizado: Barbosa (2016)

$\mathrm{Na}$ tabela 4, temos as categorias dos objetos representacionais do grupo de ensino de geografia e representações sociais, sua respectiva quantidade de trabalhos e seu percentual. Ao verificarmos os dados contidos nessa tabela, constatamos que existe um maior percentual de objetos representacionais na categoria de temas geográficos com 50\% (11 trabalhos); 
em seguida, temos a categoria processos educacionais com 31,5\% (7 trabalhos) e; o menor índice está representado pela categoria conceitos geográficos com 18,2\% (4 trabalhos).

É interessante perceber que há um grande número de objetos que podem ser abordados pela Teoria das Representações Sociais nesse grupo temático. Em geral, os grandes temas da Geografia causam certa estranheza entre os educandos em virtude da dificuldade no equacionamento de problemas gerados pela vivência humana na Terra. São temas polêmicos e propícios para a construção de representações sociais.

No que se refere aos resultados do segundo grupo temático, ciência geográfica e representações sociais, também realizamos uma categorização em relação a esse quesito pesquisado. Nesse caso, foram utilizados como categorias os ramos da Ciência Geográfica (Geografia Física e Geografia Humana).

Tabela 5- Categorização dos objetos representacionais do grupo temático ciência geográfica e representações sociais

\begin{tabular}{c|c|c|c} 
Categoria & $\begin{array}{c}\text { Frequência } \\
\left(\mathbf{n}^{\circ} \text { absoluto }\right)\end{array}$ & $\begin{array}{c}\text { Porcentagem } \\
\mathbf{( \% )}\end{array}$ & $\begin{array}{c}\text { Objetos representacionais } \\
\text { (exemplos encontrados) }\end{array}$ \\
Geografia Humana & 18 & 75 & $\begin{array}{c}\text { Moradia } \\
\text { Reforma agrária } \\
\text { Bairro Bugio } \\
\text { Cidade desenvolvida }\end{array}$ \\
\hline Geografia Física & 6 & 25 & Programa minha casa minha vida \\
\hline Total & 24 & 100 & Processo de arenização \\
& & & Caverna \\
& & & Seca \\
\hline
\end{tabular}

Fonte: BNTD (2016)

Organizado: Barbosa (2016)

Nesse grupo temático, observado na Tabela 5, temos um grande número de pesquisas cujo objeto representacional tem acento na Geografia Humana (75\%). No tocante à Geografia Física, temos uma quantidade bem menor, somente com $25 \%$ das produções. Esses dados revelam que possivelmente os pesquisadores da Geografia Humana utilizam mais a teoria das representações sociais do que os pesquisadores da Geografia Física. Mas revela, também, a capacidade de abrangência da Teoria das Representações Sociais, pois esta 
auxilia na compreensão de que os fenômenos físicos são apreendidos de modo diferente pelos diversos grupos sociais e isso implica na análise de como organizar projetos de uso ou preservação de elementos da natureza.

O terceiro critério de análise dos dados corresponde à metodologia de pesquisa utilizada nesses trabalhos. Buscamos conhecer os caminhos que foram percorridos nessas investigações. Quanto aos instrumentos de coleta de dados, os pesquisadores, em todos os grupos temáticos, fizeram uso de uma variedade deles, conforme podemos observar a seguir.

Tabela 6 - Instrumentos utilizados nos trabalhos do grupo temático de ensino de geografia e representação social

\begin{tabular}{l|c} 
INSTRUMENTOS & $\mathbf{N}^{\circ}$ DE TRABALHOS \\
\hline Questionários & 12 \\
\hline Entrevistas & 9 \\
\hline TALP & 6 \\
\hline Desenhos & 2 \\
\hline Grupo focal & 2 \\
\hline Observação direta & 2 \\
\hline Produção de texto & 2 \\
\hline Fotografia & 1 \\
\hline Mapas mentais & 1 \\
\hline
\end{tabular}

Fonte: BNTD (2016)

Organizado: Barbosa (2016)

A tabela 6 nos revela que no grupo temático ensino de geografia e representações sociais, o recurso mais utilizado foi o questionário que aparece em 12 (doze) investigações. Em seguida, temos as entrevistas, presentes em 9 (nove) trabalhos e o Teste de Associação Livre de Palavras - TALP em 6 (seis) investigações. Em uma menor escala percebemos o uso de desenhos, grupos focais, observação direta e produção textual que aparecem cada um em 2 (duas) pesquisas. A fotografia e os mapas metais aparecem em 1 (um) trabalho cada. Com relação aos instrumentos utilizados no grupo temático ciência geográfica e representações sociais, temos a tabela 7 .

\footnotetext{
4 O Teste de Associação Livre de Palavras também conhecido como TALP é um instrumento utilizado para coleta de dados através da associação de palavras realizada pelos participantes a partir de uma expressão indutora previamente estabelecida e apresentada pelo pesquisador (SÁ, 1996).
} 
Tabela 7 - Instrumentos utilizados nas pesquisas do grupo temático da ciência geográfica e representações sociais

\begin{tabular}{c|c}
\hline INSTRUMENTOS & $\mathbf{N}^{\circ}$ DE TRABALHOS \\
\hline Entrevistas & 20 \\
\hline Questionários & 10 \\
\hline Observação direta & 8 \\
\hline TALP & 5 \\
\hline Análise de jornais e revistas & 4 \\
\hline Análise de filme & 1 \\
\hline Fotografia & 1 \\
\hline Mapas mentais & 1 \\
\hline
\end{tabular}

Fonte: BNTD (2016)

Organizado: Barbosa (2016)

Com referência à tabela 7 , observamos que no grupo temático da ciência geografia e representação social, o instrumento mais usual foi a entrevista presente em 20 (vinte) investigações; o questionário é utilizado 10 (dez) vezes e a observação direta está presente em 8 (oito) trabalhos. Temos também o TALP presente em 5 (cinco) trabalhos e a análise de jornais e revistas em 4 (quatro). Em número menor temos o uso de mapas mentais, fotografia e análise de filme, presentes 1 (uma) única vez.

Realizando um panorama geral em relação aos recursos utilizados nas pesquisas, constatamos que o questionário e a entrevista são os mais utilizados. A ênfase a esses recursos acontece pelo fato destes serem bem conhecidos pelos pesquisadores e possibilitar extrair do público investigado um rico arsenal de informações. Além disso, podemos destacar também a presença em todos os grupos do TALP que é um instrumento utilizado para coleta de dados em representações sociais dentro da perspectiva da Teoria do Núcleo Central.

A variedade de instrumentos de coleta de dados revela um aspecto que marca a teoria desde o seu nascedouro, ou seja, não há uma metodologia privilegiada pela Teoria. Podese fazer uso dos métodos de coleta e análise de dados já consagrados pelas ciências sociais. Portanto, é o olhar do pesquisador embasado pela teoria que irá observar a presença dos conteúdos representacionais nos dados coletados. 


\section{CONSIDERAÇÕES FINAIS}

Ao considerar os dados que foram coletados, inicialmente afirmamos que o uso da teoria das representações sociais nas pesquisas geográficas são permeadas de possibilidades, isso porque, reconhecer as representações sociais de diferentes objetos geográficos, compartilhadas pelos indivíduos nos grupos sociais nos apresenta um diagnóstico de como essa ciência é vista nesses diferentes grupos. Além disso, podemos visualizar que esse aporte teórico pode ser utilizado nos diferentes ramos da geografia, seja pesquisas voltadas para educação geográfica, seja para temas mais específicos humanos e/ou físicos, como pode ser visto na diversidade de objetos representacionais visualizados nesta investigação.

Com relação aos instrumentos de coleta de dados, temos como destaque o uso de questionário e entrevista, que são instrumentos bem conhecidos pelos pesquisadores de Geografia, contudo, pela plasticidade da teoria, observamos que há uma diversidade de instrumentos que podem ser utilizados para as investigações com interesse em representação social. É importante fazer uma ressalva que a teoria não impõe o uso específico de nenhum instrumento, assim, eles vão depender do objeto e dos objetivos da pesquisa, estabelecidos por cada pesquisador.

Ao observar a espacialização desses trabalhos destacamos que para o grupo temático do ensino de geografia, os polos de pesquisas com maior destaque são as regiões Nordeste e Sul. Em relação ao grupo da ciência geográfica, temos as regiões Sudeste e Sul.

Observamos que o volume de produção anual dos últimos anos nos dois grupos temáticos teve um aumento significativo, porém, é importante ressaltar que há muitos objetos geográficos e campos de pesquisa possíveis de serem explorados através da teoria das representações sociais no país e, dada a importância da Teoria para a compreensão desses objetos e, consequentemente, uma ampliação do conhecimento sobre eles, recomendamos uma maior divulgação desses trabalhos. 


\section{REFERÊNCIAS}

1. ABRIC, Jean-Claudeb. O estudo experimental das representações sociais. In.: JODELET, Denise (Org.). As representações sociais. Rio de Janeiro: EdUERJ, 2001.

2. BONFIM, Natanael Reis. A representação social como teoria e método. BONFIM, Natanael Reis; ROCHA, Lurdes Bertol (Orgs). As representações na Geografia. Ilheus-BA: Editus, 2012. P. 13-30.

3. CAVALCANTI, Lana de Sousa. Geografia, escola e construção de conhecimentos. Campinas: Papirus, 1998.

4. JODELET, Denise. Representações Sociais: um domínio em expansão. In. JODELET, Denise. (Org.) As representações sociais. Tradução: Lilian Ulup. Rio de Janeiro: EDUERJ. 2001. p. 19-44.

5. MARTINS, Alberto Mesaque; CARVALHO, Cristiene Adriana da Silva; ANTUNES-ROCHA, Maria Isabel. Pesquisa em representações sociais no Brasil: cartografia dos grupos registrados no CNPq. Revista Psicologia: Teoria e Prática, São Paulo, v. 16, n. 1, p. 104-114, jan./abr., 2014.

6. MOSCOVICI, Serge. A psicanálise, sua imagem e seu público. Tradução de Sonia Fuhrmann. Petrópolis: Vozes, 2012.

7. MOSCOVICI, Serge. Das representações coletivas às representações sociais: elementos para uma história. In: JODELET, Denise (Org). As representações sociais. Tradução de Lilian Ulup. Rio de janeiro: EdUERJ. 2001. p. 45-66.

8. MOSCOVICI, Serge. Representações sociais: investigações em psicologia social. Petrópolis: Vozes, 2003.

9. SÁ, Celso Pereira de. A construção do Objeto de Pesquisa em Representações Sociais. Rio de Janeiro: EdUERJ, 1998.

10. SÁ, Celso Pereira de. Núcleo Central das Representações Sociais. Petrópolis-RJ: Vozes, 1996.

11. FERREIRA, Norma Sandra de Almeida. As pesquisas denominadas "estado da arte". Educação \& Sociedade, Campinas, ano XXIII, no. 79, p. 257-272, 2002. Disponível em: <http://www. scielo.br/pdf/es/v23n79/10857.pdf> Acesso em: 05 ago. 2016.

12. FONSECA, Ricardo Lopes; SALVI, Rosana Figueiredo; TORRES, Eloiza Cristiane. O Estado da arte das pesquisas em dissertações de mestrado em ensino de geografia: Contribuição para uma avaliação a partir das pesquisas nos programas nacionais de Stricto Sensu (2008 - 2012). Revista da Associação Nacional de Pós-graduação e Pesquisa em Geografia (Anpege). p.141 163, V.10, n.13, jan-jun.2014. Disponível em: < http://anpege.org.br/revista/ojs-2.4.6/index. php/anpege08/article/view/331/184> Acesso em: 03 ago. 2016.

ARTIGO RECEBIDO EM NOVEMBRO DE 2016

ARTIGO APROVADO EM ABRIL DE 2017 